\title{
An Extensive Cholesteatoma with Bezold's Abscess
}

\author{
Norhafiza Mat Lazim¹, Asma Abdullah ${ }^{2}$ \\ ${ }^{1}$ ORL-HNS Department, HUSM Kubang Kerian, Kota Bharu, Malaysia; ${ }^{2}$ Head of Department, ORL Head \& Neck Surgery, UKM \\ Medical Centre, Jalan Ya’akob Latif, Kuala Lumpur, Malaysia. \\ Email: norhafiza@kck.usm.my, asmaent@yahoo.com
}

Received March 20 ${ }^{\text {th }}$, 2011; revised April 23 ${ }^{\text {rd }}, 2011$; accepted May $6^{\text {th }}, 2011$.

\begin{abstract}
Cholesteatoma has been known to be associated with multiple complications either extracranially or intracranially. Among the extracranial complications, mastoiditis and mastoid abscess are the most common. Bezold's abscess formation with cholesteatoma is a rare occurrence but when present can lead to sinister sequalae if not properly managed. The treatment of cholesteatoma is mainly by surgical exploration namely mastoidectomy. The aim of treatment is to eradicate the diseased mastoid and to prevent subsequent complications. Beside surgical intervention, the patient will also require intensive systemic and topical antibiotic therapy. With proper treatment patient will be hindered from experiencing unwanted complications.
\end{abstract}

Keywords: Cholesteatoma, Bezold's Abscess, Mastoidectomy

\section{Introduction}

Cholesteatoma is a benign disease which affects various spaces such as middle ear, mastoid or petrous bone. It is known for its tendency to recur. It has capacity for progressive and independent growth and causes bony erosion. If not properly managed it inevitably will result in complications. These complications can be divided into extracranial and intracranial. The extracranial complications are mastoid abscess, subperiosteal abscess, petrositis, labyrinthine fistula and Bezold's abscess. Intracranial complications range from meningitis, subdural abscess, lateral sinus thrombosis and extradural abscess [1]. Among these complications, Bezold's abscess is scarcely reported in the literature. The reason being due to the widespread use of antibiotics in this modern antibiotic era which has exclusively prevented the forementioned complications. We report a case of a young lady presented with cholesteatoma complicated with Bezold's abscess.

\section{Case Report}

A 25 year old Chinese lady with past history of chronic otitis media since childhood presented to a private clinic with history of left ear discharge for a four month duration. It was associated with post auricular swelling. The swelling was initially small in size but has gradually in- creased over few weeks. Her pain was relieved intermittently with analgaesics. At the same time, she also had reduced hearing on the same side which worsened over few weeks prior to the presentation. She had facial asymmetry three days prior to the admission. She denied any history of vertigo or tinnitus, but there was history of intermittent low grade fever. There was no history of headache, blurring of vision or vomiting. According to her she attended a local general practitioner for few times and was prescribed with different antibiotics. Despite the topical eardrops and antibiotics, her condition did not improve. As far as past history is concerned, she had history of otorrhoea during childhood which was settled. There was no other significant history.

On clinical examination, the patient was febrile. There was left sided facial nerve palsy grade II (HouseBrackman grading system). Ear examination showed postauricular swelling over the left mastoid region which was tender on palpation. The swelling measured $3.0 \mathrm{~cm} \times$ $3.0 \mathrm{~cm} \times 4.0 \mathrm{~cm}$. The overlying skin was mildly inflamed and tender. However there was no discharge noted. Otoscopic examination revealed sagging of posterior wall of left external auditory canal. Left tympanic membrane was not visualized due to oedematous external auditory canal. Right ear was normal. There was no palpable neck node. Cranial nerves were all intact except the facial 


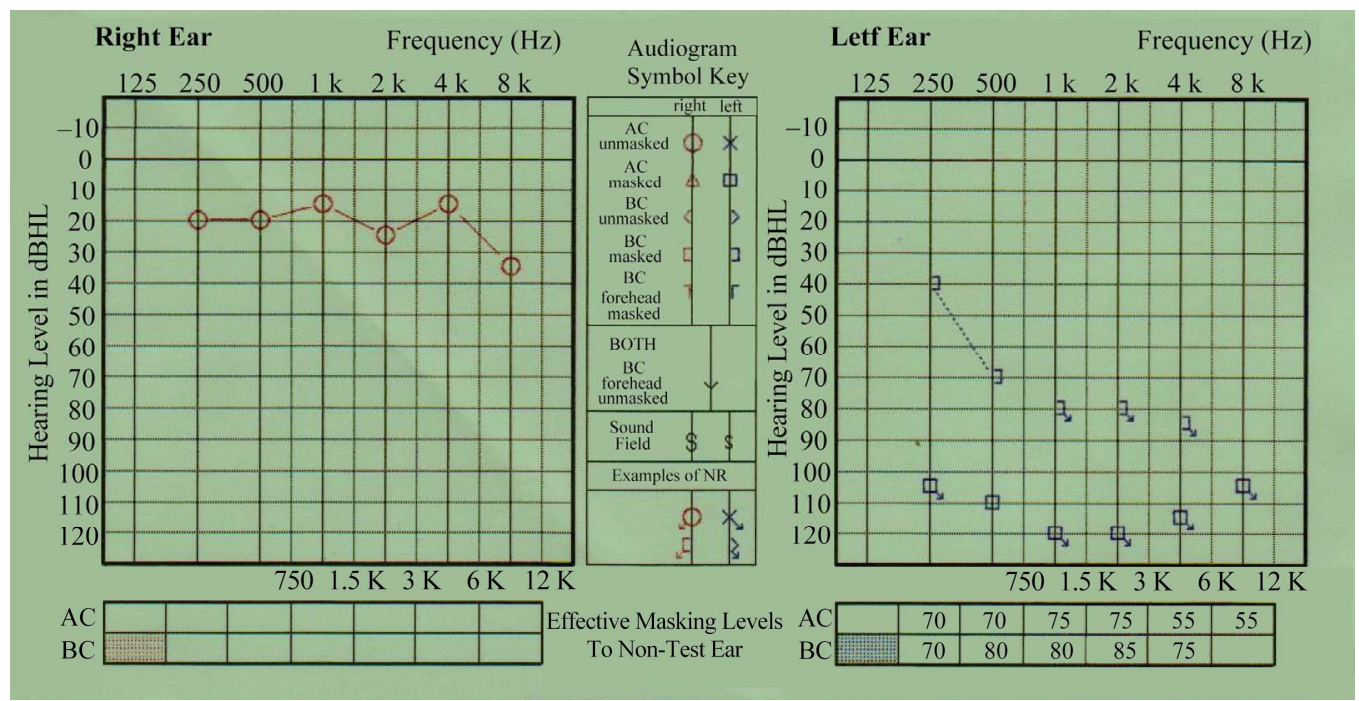

Figure 1. Pure Tone Audiogram shows profound sensorineural hearing loss of left ear and normal hearing on right ear.

nerve and the vestibulocochlear nerve. There was no nystagmus and no neck stiffness. Rinne's test was positive on the right ear and negative on the left ear with Weber's test lateralised to right ear.

Pure Tone Audiogram showed profound sensorineural hearing loss on the left ear (Figure 1). CT Scan of temporal bone from previous private hospital revealed presence of well defined mass occupying the mastoid cavity with extensive bony erosion and loss of ossicles and semicircular canals (Figure 2). The mass has also extended inferiorly to the level of C2 vertebrae. It lies inferior to sternocleidomastoid muscle (Figure 3).

The patient was admitted and started on IV Rocephine 1.0 gm twice daily. She was put under emergency list for left mastoid exploration. The procedure and risk of facial

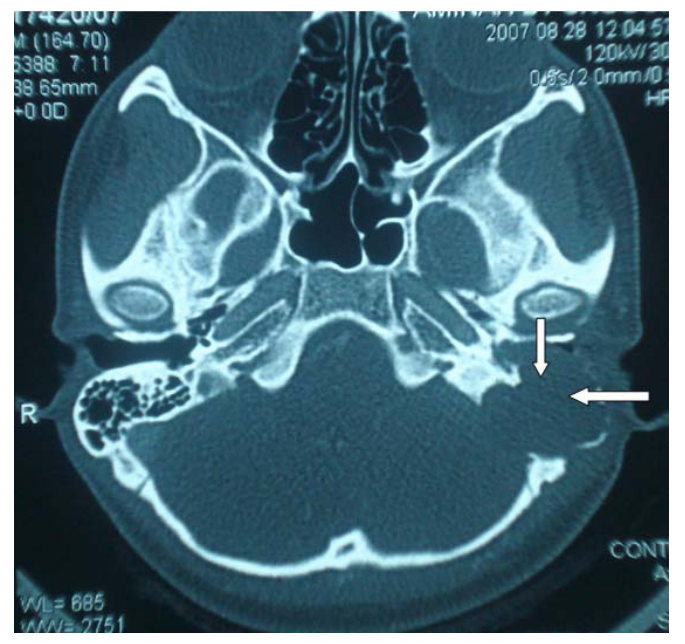

Figure 2. Axial CT Scan of temporal bone shows an extensive cholesteatoma with bone erosion. All the ossicles and semicircular canals are absent (arrow). nerve injury was explained to the patient. Intraoperative findings revealed an extensive cholesteatoma in the entire external auditory canal with erosion of the posterior wall. The pus was seen in the mastoid cavity when sternocleidomastoid was pressed. Tip of mastoid, zygomatic root, ossicles, part of tegmen tympani, part of floor of hypotympanum and part of petrous bone were all eroded. The left facial nerve was exposed from tympanic segment to two-third of vertical segment. Post operatively patient recuperating well with minimal left ear discharge. Her hearing otherwise did not improve post operatively. However, her facial nerve palsy has resolved completely.

\section{Discussion}

Cholesteatoma is a disease that can occur in middle ear, mastoid bone or petrous temporal bone. It is character-

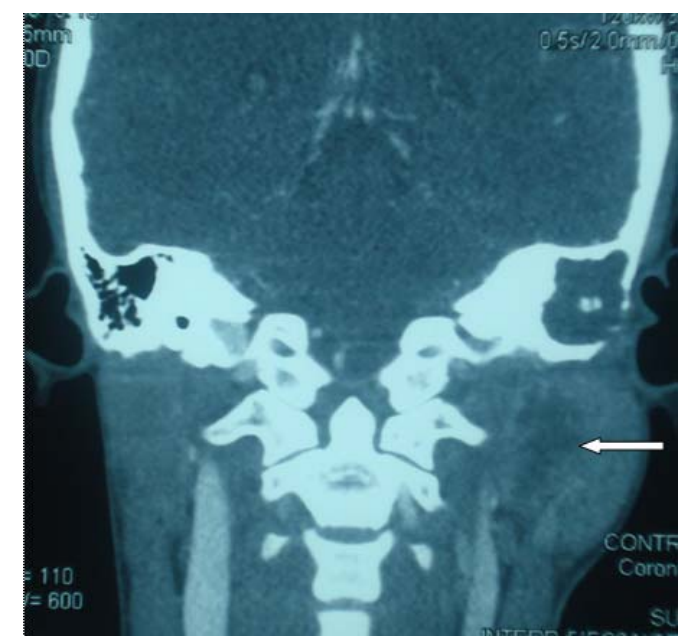

Figure 3. Coronal CT Scan of neck shows collection under left sternocleidomastoid muscle (arrow). 
ized by a tendency for bone erosion and recurrence. Once established in the middle ear, mastoid or petrous bone, cholesteatoma is destructive lesion that gradually expands and destroys adjacent structures leading to complications [2]. These complications include subperiosteal abscess, mastoid abscess, petrositis, labyrinthitis and facial nerve palsy. Intracranial complications ranging from meningitis, brain abscess, lateral sinus thrombosis and extradural abscess [3]. Bezold's abscess however is a rare complication of cholesteatoma.

Bezold's abscess occur infrequently nowadays due to the advent of antibiotics and early surgical intervention. It is defined as a collection of abscess deep to sternocleidomastoid muscle. It was introduced by a German otologist, Friedrich Bezold in 1881. Bezold distinguished this form of abscess from other more common forms, such as the subperiosteal abscess, which arise from the erosion of the outer surface of the mastoid cortex [1]. In Bezold's abscess the pus discharge escapes via a perforation of the inner side of mastoid process which then tracks down along the fascia planes of the digastrics or sternocleidomastoid muscle in the neck.

The pathogenesis of the Bezold's abscess has been attributed to the degree of pneumatisation of the mastoid bone. In a well pneumatised mastoid bone, the spaces with the thin bone can easily act as a pathway for a disease process to spread through it. In the absence of pneumatisation, the mastoid bony walls are thick and hinder the erosion process [4]. As in our case, massive cholesteatoma in the middle ear can certainly lead to bony erosion of the mastoid tip with subsequent development of the false track which acts as a conduit for the abscess to track down through the fascia plane inferiorly down to the neck.

The presence of cholesteatoma debris in the chronically infected mastoid may obstruct the infectious foci into external auditory canal and allows the foci to find a weak point in the mastoid tip [5]. The more devastating sequalae can arise when infection spread downward along great vessels to reach the perivisceral space, larynx or mediastinum. It can also descend along the intervertebral muscle to reach the retropharyngeal space. Alternatively, it could track down along the wall of subclavian artery to reach the posterior triangle of the neck and axilla or reach the suprasternal space and crosses to the the contralateral neck with more hazardous complications [5].

Clinical presentations vary and include pyrexia, otalgia, neck swelling, otorrhoea, neck pain, restriction of neck movements, facial nerve palsy and hypoacusia [6]. In the early phase of abscess formation, the sign probably was subtle and there should be a high index of suspicion in treating patients belonging to this group. The organism that is most commonly cultured is Streptococcus. Gram positive cocci and gram negative cocci as well as anaerobes have also been implicated. Other organism such as Proteus mirabilis, Staph aureus, Proteus vulgaris have also been isolated [2].

In our patient there was no risk factor for her to develop such complications. We thought that her condition worsened because of inadequate antibiotic treatment she received previously, and a very much delay in presentation to our care. But with disease clearance by radical mastoidectomy, her condition improved significantly.

CT Scan of the temporal bone and neck is the main imaging modality for diagnosis of Bezold's abscess. The findings include the presence of fluid filled middle ear and mastoid and demineralisation of the mastoid trabeculae [5]. CT Scan of neck showed the collection is inferior to mastoid but not below the level of cricoid cartilage together with obliteration of the fascia and fat plane, reticulation of the subcutaneous tissues and thickening of the skin overlying sternocleidomastoid muscle [7]. These features are seen in the CT Scan film of this case.

\section{REFERENCES}

[1] Y. Uchida, H. Ueda and T. Nakashima, "Bezold's Abscess Arising with Recurrent Cholesteatoma 20 Years after Surgery: With a Review of the 18 Cases Published in Japan since 1960," Auris Nasus Larynx, Vol. 29, No. 4, 2002, pp. 375-378. doi:10.1016/S0385-8146(02)00057-3

[2] A. Mustaffa, A. Heta, B. Kastrati, et al., "Complication of Chronic Otitis Media with Cholesteatoma during a Ten Year Period in Kosovo," European Archives of Otorhinolaryngology, Vol. 265, No. 12, 2008, pp. 1477-1482. doi:10.1007/s00405-008-0707-8

[3] S. Y. Yeh and P. W. Cheng, "Concurrent Intracranial and Extracranial Complications Secondary to Cholesteatoma: A Case Report,” Otolaryngology Head and Neck Surgery, Vol. 128, No. 1, 2003, pp.163-164. doi: $10.1067 / \mathrm{mhn} .2003 .75$

[4] B. McMullan, "Bezold's Abscess: A Serious Complication of Otitis Media,” Journal of Paediatrics and Child Health, Vol. 45, No. 10, 2009, pp. 616-618. doi:10.1111/j.1440-1754.2009.01575.x

[5] G. Marioni, C. D. Fillipis, A. Tregnaghi, R. M. Ragona and A. Stafferi, "Bezold's Abscess in Children: Case Report and Review in Literature," International Journal of Paediatric Otorhinolaryngology, Vol. 61, No. 2, 2001, pp. 173-177.

[6] P. Zappata, D. H. Chi and F. Russel, "A Unique Case of Bezold's Abscess Associated with Multiple Dural Sinus Thrombosis,” Laryngoscope, Vol. 111, No. 11, 2001, pp. 1944-1947. doi:10.1097/00005537-200111000-00013

[7] M. Castillo, V. S. Albernaz, S. K. Mukherji, M. M. Smith and J. L. Weissman, "Imaging of Bezold's Abscess," American Journal of Roentgenology, Vol. 171, No. 6, 1998, pp. 1491-1495. 